\title{
A Problem in Thermoelasticity with and without Energy Dissipation
}

\section{Khedr Mona El M ${ }^{1}$ and Khader SE**}

${ }^{1}$ Department of Physics, Faculty of Science University of Alexandria, Egypt

${ }^{2}$ Department of Mathematical and Theoretical Physics Atomic Energy Authority, Egypt

\begin{abstract}
In this work, we are compared between the theory of thermoelasticity with two relaxation times and the theory of thermoelasticity without energy dissipation. To a half-space overlaid by a thick layer of a different material. The upper surface of the layer is taken to be traction free and is subjected to a constant thermal shock. We are used Laplace transform to eliminate the time variable t. The inverses Laplace transforms are obtained by using a numerical method based on Fourier expansion techniques comparison between the predictions of both theories are discussed. Numerical results are computed for the temperature, displacement and stress distributions. The numerical results are represented graphically.
\end{abstract}

Keywords: GL theory; GN theory; Laplace transform; Half-space; Different material

\section{Introduction}

Thermoelasticity's importance is due to its many applications in diverse fields such as geophysics, plasma physics and related topics, especially in the design of nuclear reactors.

Lord and Shulman [1] introduced the theory of generalized thermoelasticity with one relaxation time for the special case of an isotropic body. In this theory, the new equation of heat was obtained by using a new law of heat conduction instead of Fourier's law. The heat equation associated with this theory is hyperbolic and hence eliminates the above mentioned defect. The equation of motion is the same as that of the coupled theory of thermoelasticity. Sherief and Anwar [2] solved a generalized thermoelasticity problem for a plate subjected to moving heat sources on both sides. Sherief and Khader [3] discussed the propagation of discontinuities in electromagneto generalized thermoelasticity in cylindrical regions. Chen et al. [4] studied the Transient thermal stresses in a multilayered anisotropic medium. Sharma et al. [5] discussed the Reflection of generalized thermoelastic waves from the boundary of a half-space. Elhagary [6] solved the problem of a two-dimensional problem for two media in the generalized theory of thermoelasticity. Abd El-Latief and Khader [7] studied the fractional model of thermoelasticity for a half-space overlaid by a thick layer. Sherief and El-Maghraby [8] solved the problem of a thick plate in the theory of generalized thermoelastic diffusion.

Green and Lindsay [9] introduced two different lag times in the stress- strain relations and the entropy expression. This theory is known as the theory of thermoelasticity with two relaxation times. In this theory both the equations of motion and of heat conduction are hyperbolic. The equation of motion differs from that of the coupled thermoelasticity theory. Sinha and Elsibai [10] discussed the reflection of thermoelastic waves at a solid half-space with two thermal relaxation times. Sherief $[11,12]$ solved a state space approach to thermoelasticity with two relaxation times and the fundamental solution for thermoelasticity with two relaxation times and a thermo-mechanical shock problem for thermoelasticity with two relaxation times. Anwar and Sherief [13] studied the boundary integral equation formulation for thermoelasticity with two relaxation times. Song et al. [14] solved the transient disturbance in a half space under thermoelasticity with two relaxation times due to moving internal heat source.

The theory of thermoelasticity without energy dissipation (GN theory) was proposed by Green and Naghdi [15]. The most important aspect of this theory, which is not present in other thermoelasticity theories, is that this theory does not accommodate dissipation of thermal energy. Abd El-Latief and Khader [16] obtained the exact solution of thermoelastic problem for a one-dimensional bar without energy Dissipation. Quintanilla $[17,18]$ discussed the spatial behavior in thermoelasticity without energy dissipation and existence in thermoelasticity without energy dissipation. Iesan [19] discussed the theory of thermoelasticity without energy dissipation. Verma and Hasebe [20] solved the dispersion of thermoelastic waves in a plate with and without energy dissipation. Marin and Baleanu [21] discussed the vibrations in thermoelasticity without energy dissipation for micropolar bodies.

\section{Formulation of the Problem}

We consider a problem for a half-space overlaid by a thick layer of a different material within the context of the theory of thermoelasticity with two relaxation times and the theory of thermoelasticity without energy dissipation. The $\mathrm{x}$-axis is taken perpendicular to the surfaces of the half-space and of the layer. The layer occupies the region $0 \leq x \leq L$, while the half-space occupies the region $x>L$. The upper surface of the layer is taken to be traction free and is subjected to a constant thermal shock.

Due to the physics of the problem, all the functions considered will depend on $\mathrm{x}$ and $\mathrm{t}$ only. The displacement components have the form

$$
u_{x=} u_{i}(x, t), u_{y}=u_{z}=0, i=1,2 .
$$

From now on the suffix 1 denotes quantities in the region of the layer while the suffix 2 denotes quantities in the region of the half-space.

\section{Solution for GL-Theory}

The equations of motion have the form

*Corresponding author: Khader SE, Department of Mathematical and Theoretica Physics Atomic Energy Authority, Egypt, Tel:15151545; E-mail: shabolaaaa@yahoo.com

Received August 20, 2017; Accepted August 30, 2017; Published September 04, 2017

Citation: Khedr Mona El M, Khader SE (2017) A Problem in Thermoelasticity with and without Energy Dissipation. J Phys Math 8: 243. doi: 10.4172/20900902.1000243

Copyright: (C) 2017 Khedr Mona El M, et al. This is an open-access article distributed under the terms of the Creative Commons Attribution License, which permits unrestricted use, distribution, and reproduction in any medium, provided the original author and source are credited. 
Citation: Khedr Mona El M, Khader SE (2017) A Problem in Thermoelasticity with and without Energy Dissipation. J Phys Math 8: 243. doi: 10.4172/2090-0902.1000243

Page 2 of 5

$$
\left(\lambda_{i}+2 i_{i}\right) \frac{\partial^{2} u_{i}}{\partial x^{2}}-\tilde{a}_{i}\left(1+v_{i} \frac{\partial}{\partial t}\right) \frac{\partial T_{i}}{\partial x}=\rho_{i} \frac{\partial^{2} u_{i}}{\partial t^{2}}, i=1,2
$$

The generalized equation of heat conduction for both media are given by

$$
k_{i} \frac{\partial^{2} T}{\partial x^{2}}=\rho_{i} c_{E_{i}}\left(1+\tau_{i} \frac{\partial}{\partial t}\right) \frac{\partial T_{i}}{\partial t}+\gamma_{i} T_{0} \frac{\partial^{2} u_{i}}{\partial x \partial t} i=1,2
$$

In the above equations $\lambda_{i}, \mu_{i}$ are Lamés constants, $\rho_{i}$ is the density, $\tau_{i}, v_{i}$ are the two relaxation times, $k_{i}$ is the thermal conductivity, $c_{E \mathrm{i}}$ is the specific heat at constant strain, $T_{i}$ is the absolute temperature and $T_{0}$ is a reference temperature assumed to be such that $/\left(T_{i}-T_{0}\right) / T_{0} I<<1, \gamma_{i}$ a material constant given by $\gamma_{i}=\left(3 \lambda_{i}+2 \mu_{i}\right) \alpha_{i}$ where $\alpha_{i}$ is the coefficient of linear thermal expansion.

The constitutive equations have the form:

$$
\sigma_{i}=\left(\lambda_{i}+2 \mu_{i}\right) \frac{\partial u_{i}}{\partial x}-\gamma_{i}\left(T_{i}-T_{0}+v_{i} \frac{\partial T_{i}}{\partial t}\right)
$$

where $\sigma_{i}$ denotes the stress component $\sigma_{x x}$ in medium i.

We also have the Fourier's law of heat conduction, namely

$q_{i}=-k_{i} \frac{\partial T_{i}}{\partial x}$

where $q_{i}$ is the heat flux in $x$-direction in medium $i$.

Let us introduce the following non- dimensional variables,

$x^{*}=c_{1} \eta_{1} x, u_{i}^{*}=c_{1} \eta_{1} u_{i}, t^{*}=c_{1}^{2} \eta_{1} t, \tau_{i}^{*}=c_{1}^{2} \eta_{1} \tau_{i}, \quad v_{i}^{*}=c_{1}^{2} \eta_{1} v_{i}$,

$$
\sigma_{i}^{*}=\frac{\sigma_{i}}{\left(\lambda_{1}+2 \mu_{1}\right)}, \theta_{i}^{*}=\frac{\gamma_{1}\left(T_{i}-T_{0}\right)}{\left(\lambda_{1}+2 \mu_{1}\right)}, q_{i}^{*}=\frac{\gamma_{1}}{k_{1} c_{1} \eta_{1}\left(\lambda_{1}+2 \mu_{1}\right)} q_{i},
$$

where $c_{1}=\sqrt{\frac{\lambda_{1}+2 \mu_{1}}{\rho_{1}}}, \eta_{1}=\frac{\rho_{1} c_{E 1}}{k_{1}}$

The governing equations (1-4) in non-dimensional form become (dropping the asterisks for convenience)

$$
\begin{aligned}
& \psi_{i} \frac{\partial^{2} u_{i}}{\partial x^{2}}-\left(1+v_{i} \frac{\partial}{\partial t}\right) \frac{\partial \theta_{i}}{\partial x}=\xi_{i} \frac{\partial^{2} u_{i}}{\partial t^{2}} \\
& \frac{\partial^{2} \theta_{i}}{\partial x^{2}}=\delta_{i}\left(1+\tau_{i} \frac{\partial}{\partial t}\right) \frac{\partial \theta_{i}}{\partial t}+\varphi_{i} \frac{\partial^{2} u_{i}}{\partial x \partial t} \\
& \sigma_{i}=\frac{\gamma_{i}}{\gamma_{1}}\left[\psi_{i} \frac{\partial u_{i}}{\partial x}-\left(\theta_{i}+v_{i} \frac{\partial \theta_{i}}{\partial t}\right)\right] \\
& q_{i}=-\alpha_{i} \frac{\partial \theta_{i}}{\partial x}
\end{aligned}
$$

where,

$\psi_{i}=\frac{\gamma_{1}\left(\lambda_{i}+2 \mu_{i}\right)}{\gamma_{i}\left(\lambda_{1}+2 \mu_{1}\right)}, \xi_{i}=\frac{\gamma_{1} \rho_{i}}{\gamma_{i} \rho}, \delta_{i}=\frac{\mu_{i}}{\mu_{1}}, \varphi_{i}=\frac{\gamma_{i} T_{0} \gamma_{1}}{k_{1}\left(\lambda_{1}+2 \mu_{1}\right) \eta_{1}}, \alpha_{i}=\frac{k_{i}}{k_{1}}$

The boundary conditions of the problem can be written as:

$\sigma_{1=} 0$, at $x=0$

$\theta_{1}=f(t)$, at $x=0$

The continuity conditions between the two media are:

$u_{1}=u_{2}, \theta_{1}=\theta_{2}, \sigma_{1}=\sigma_{2}, q_{1}=q_{2}$ at $x=L$

We assume also that the initial conditions of a problem are homogeneous.
Applying the Laplace transform with parameter s defined by the relation

$$
\bar{f}(s)=\int_{0}^{\infty} f(t) e^{-s t} d t
$$

to both sides of equations 5-8, we obtain

$$
\begin{aligned}
& \left(\psi_{i} \frac{\partial^{2}}{\partial x^{2}}-\xi_{i} s^{2}\right) \bar{u}_{i}=\left(1+v_{i} s\right) \frac{\partial \bar{\theta}_{i}}{\partial x} \\
& {\left[\frac{\partial^{2}}{\partial x^{2}}-\delta_{i}\left(1+\tau_{i} s\right) s\right] \bar{\theta}_{i}=\varphi_{i} s \frac{\partial \bar{u}_{i}}{\partial x}} \\
& \bar{\sigma}_{\iota}=\frac{\gamma_{i}}{\gamma_{1}}\left[\psi\left(\frac{\partial \bar{u}_{i}}{\partial x}-\left(1+v_{i} s\right) \bar{\theta}_{i}\right]\right. \\
& \bar{q}_{i}=-\alpha_{i} \frac{\partial \bar{\theta}_{i}}{\partial x}
\end{aligned}
$$

Eliminating $\bar{\theta}_{i}$ between equations (12) and (13), we get the following fourth order differential equation satisfied by $\bar{u}_{i}$ :

$$
\left\{\psi_{i} D^{4}-D^{2}\left[\psi_{i} \delta_{i}\left(1+\tau_{i} s\right) s+\xi_{i} s^{2}+\varphi_{i}\left(1+v_{i} s\right) s\right]+\xi_{i} s^{3} \delta_{i}\left(1+\tau_{i} s\right)\right\} \bar{u}_{i}=0
$$

where $D=\frac{\partial}{\partial x}$

Solving equation (16), we obtain for the two different media,

$$
\begin{aligned}
& \bar{u}_{1}=\left(1+v_{1} s\right)\left[A_{1} k_{11} e^{k_{11} x}+A_{2} k_{12} e^{k_{12} x}-A_{3} k_{11} e^{-k_{11} x}-A_{4} k_{12} e^{-k_{12} x}\right] \\
& \bar{u}_{2}=-\left(1+v_{2} s\right)\left[k_{21} A_{5} e^{-k_{21} x}+k_{22} A_{6} e^{-k_{22} x}\right]
\end{aligned}
$$

where $A_{1}, A_{2}, A_{3}, A_{4}, A_{5}$ and $A_{6}$ are parameters that depend on $s$ only and $k_{i 1}, k_{i 2}$ are the roots with positive real parts of the characteristic equation

$$
\psi_{i} k^{4}-k^{2}\left[\psi_{i} \delta_{i}\left(1+\tau_{i} s\right) s+\xi_{i} s^{2}+\varphi_{i}\left(1+v_{i} s\right) s\right]+\xi_{i} s^{3} \delta_{i}\left(1+\tau_{i} s\right)=0
$$

We note here that we have kept only the terms in equation (17b) that is bounded at infinity.

Similarly, eliminating $\bar{u}_{i}$ between equations (12), (13) we see that $\bar{\theta}$ satisfies equation 16 . Thus, the solution compatible with equation (12) is given by

$$
\begin{aligned}
& \bar{\theta}_{1}=\left(k_{11}^{2}-s^{2}\right)\left[A_{1} e^{k_{11} x}+A_{3} e^{-k_{11} x}\right]+\left(k_{12}^{2}-s^{2}\right)\left[A_{2} e^{k_{12} x}+A_{4} e^{-k_{12} x}\right] \\
& \bar{\theta}_{2}=\left(\psi_{2} k_{21}^{2}-\xi_{2} s^{2}\right) A_{5} e^{-k_{21} x}+\left(\psi_{2} k_{22}^{2}-\xi_{2} s^{2}\right) A_{6} e^{-k_{22} x} .
\end{aligned}
$$

Substituting from equations (17), (19) into equation (14), we obtain

$$
\begin{aligned}
& \bar{\sigma}_{1}=s^{2}\left(1+v_{1} s\right)\left[A_{1} e^{k_{11} x}+A_{2} e^{k_{12} x}+A_{3} e^{-k_{11} x}+A_{4} e^{-k_{12} x}\right], \\
& \bar{\sigma}_{2}=\gamma \xi_{2} \sigma^{2}\left(1+v_{2} s\right)\left[A_{5} e^{-k_{21} x}+A_{6} e^{-k_{22} x}\right],
\end{aligned}
$$

where $\gamma=\gamma_{2} / \gamma_{1}$.

Substituting from equations (19) into equation (15), we obtain the heat flux component $q_{i}$ in the form

$$
\begin{aligned}
& \bar{q}_{1}=-k_{11}\left(k_{11}^{2}-s^{2}\right)\left[A_{1} e^{k_{11} x}-A_{3} e^{-k_{11} x}\right]-k_{12}\left(k_{12}^{2}-s^{2}\right)\left[A_{2} e^{k_{12} x}-A_{4} e^{-k_{12} x}\right], \\
& \bar{q}_{2}=\alpha_{2}\left[k_{21}\left(\psi_{2} k_{21}^{2}-\xi_{2} s^{2}\right) A_{5} e^{-k_{21} x}+k_{22}\left(\psi_{2} k_{22}^{2}-\xi_{2} s^{2}\right) A_{6} e^{-k_{22} x}\right] .
\end{aligned}
$$

Applying the Laplace transform with parameter $s$ to both sides of equations (9-11), we get 
Citation: Khedr Mona El M, Khader SE (2017) A Problem in Thermoelasticity with and without Energy Dissipation. J Phys Math 8: 243. doi: 10.4172/2090-0902.1000243

Page 3 of 5

$$
\begin{aligned}
& \bar{\theta}_{1}=\bar{f}(s), \text { at } x=0, \\
& \bar{\sigma}_{1}=0 \text {, at } x=0 . \\
& \bar{u}_{1}=\bar{u}_{2}, \bar{\theta}_{1}=\bar{\theta}_{2}, \bar{\sigma}_{1}=\bar{\sigma}_{2}, \bar{q}_{1}=\bar{q}_{2} \text {, at } x=L .
\end{aligned}
$$

Substituting from equations (17), (19), (20) and (21) into equations (22)-(24), we obtain the following system of linear equations in the unknown parameters $A_{1}-A_{6}$

$$
\begin{aligned}
& A_{1}+A_{2}+A_{3}+A_{4}=0 \\
& \left(k_{11}^{2}-s^{2}\right)\left[A_{1}+A_{3}\right]+\left(k_{12}^{2}-s^{2}\right)\left[A_{2}+A_{4}\right]=\frac{\theta_{0}}{s} \\
& k_{11} A_{1} e^{k_{11} L}+k_{12} A_{2} e^{k_{12} L}-k_{11} A_{3} e^{-k_{11} L}-k_{12} A_{4} e^{-k_{12} L} \\
& +\frac{\left(1+v_{2} s\right)}{\left(1+v_{1} s\right)}\left[k_{21} A_{5} e^{-k_{21} L}+k_{22} A_{6} e^{-k_{22} L}\right]=0 \\
& \left(k_{11}^{2}-s^{2}\right)\left[A_{1} e^{k_{11} L}+A_{3} e^{-k_{11} L}\right]+\left(k_{12}^{2}-s^{2}\right)\left[A_{2} e^{k_{12} L}+A_{4} e^{-k_{12} L}\right] \\
& -\left(\psi_{2} k_{21}^{2}-\xi_{2} s^{2}\right) A_{5} e^{-k_{21} L}+\left(\psi_{2} k_{22}^{2}-\xi_{2} s^{2}\right) A_{6} e^{-k_{22} L}=0 \\
& A_{1} e^{k_{11} L}+A_{2} e^{k_{12} L}+A_{3} e^{-k_{11} L}+A_{4} e^{-k_{12} L} \\
& -\frac{\gamma \xi_{2}\left(1+v_{2} s\right)}{\left(1+v_{1} s\right)}\left[A_{5} e^{-k_{21} L}+A_{6} e^{-k_{22} L}\right]=0 \\
& k_{11}\left(k_{11}^{2}-s^{2}\right)\left[A_{1} e^{k_{11} L}-A_{3} e^{-k_{11} L}\right]+k_{12}\left(k_{12}^{2}-s^{2}\right)\left[A_{2} e^{k_{12} L}-A_{4} e^{-k_{12} L}\right] \\
& +\alpha_{2}\left[k_{21}\left(\psi_{2} k_{21}^{2}-\xi_{2} s^{2}\right) A_{5} e^{-k_{21} L}+k_{22}\left(\psi_{2} k_{22}^{2}-\xi_{2} s^{2}\right) A_{6} e^{-k_{22} L}\right]=0
\end{aligned}
$$

\section{Solution for GN-Theory}

The equations of motion have the form

$$
\left(\lambda_{i}+2 i_{i}\right) \frac{\partial^{2} u_{i}}{\partial x^{2}}-\gamma_{i} \frac{\partial T_{i}}{\partial x}=\rho_{i} \frac{\partial^{2} u_{i}}{\partial t^{2}}, i=1,2
$$

The generalized equation of heat conduction for both media are given by

$$
k_{i}^{*} \frac{\partial^{2} T}{\partial x^{2}}=\rho_{i} c_{E i} \frac{\partial^{2} T_{i}}{\partial t^{2}}+\gamma_{i} T_{0} \frac{\partial^{3} u_{i}}{\partial x \partial t^{2}}, i=1,2
$$

The constitutive equations have the form

$\sigma_{i}=\left(\lambda_{i}+2 \mu_{i}\right) \frac{\partial u_{i}}{\partial x}-\gamma_{i}\left(T_{i}-T_{0}\right)$

We also have the Fourier's law of heat conduction, namely

$q_{i}=-k_{i} \frac{\partial T_{i}}{\partial x}$

The equations (29)-(32) in non-dimensional form become

$\psi_{i} \frac{\partial^{2} u_{i}}{\partial x^{2}}-\frac{\partial \theta_{i}}{\partial x}=\xi_{i} \frac{\partial^{2} u_{i}}{\partial t^{2}}$

$C_{t i}^{2} \frac{\partial^{2} \theta_{i}}{\partial x^{2}}=\frac{\partial^{2} \theta_{i}}{\partial t^{2}}+\varphi_{i} \frac{\partial^{3} u_{i}}{\partial x \partial t^{2}}$

$\sigma_{i}=\frac{\gamma_{i}}{\gamma_{1}}\left[\psi \frac{\partial u_{i}}{i}-\theta_{i}\right]$

$q_{i}=-\alpha_{i} \frac{\partial \theta_{i}}{\partial x}$

where $C_{t i}^{2}=\frac{k_{i}^{*}}{\rho_{i} c_{E_{i}} c_{1}^{2}}$

Solution of the problem in the Laplace transform domain
Applying the Laplace transform with parameter s to both sides of equations (33)-(36), we obtain

$$
\begin{aligned}
& \left(\psi_{i} \frac{\partial^{2}}{\partial x^{2}}-\xi_{i} s^{2}\right) \bar{u}_{i}=\frac{\partial \bar{\theta}_{i}}{\partial x} \\
& {\left[C_{t i}^{2} \frac{\partial^{2}}{\partial x^{2}}-s^{2}\right] \bar{\theta}_{i}=\varphi_{i} s^{2} \frac{\partial \bar{u}_{i}}{\partial x}} \\
& \bar{\sigma}_{i}=\frac{\gamma_{i}}{\gamma_{1}}\left[\psi_{i} \frac{\partial \bar{u}_{i}}{\partial x}-\bar{\theta}_{i}\right] \\
& \bar{q}_{i}=-\alpha_{i} \frac{\partial \bar{\theta}_{i}}{\partial x}
\end{aligned}
$$

Eliminating $\bar{\theta}_{i}$ between equations (37), (38), we get the following fourth order differential equation satisfied by $\bar{u}_{i}$

$$
\left\{\psi_{i} C_{t i}^{2} D^{4}-D^{2}\left[\psi_{i} s^{2}+C_{t i}^{2} \xi_{i} s^{2}+\varphi_{i} s^{2}\right]+\xi_{i} s^{4}\right\} \bar{u}_{i}=0
$$

The solution to equation (41) is again of the form in equation (16)

$$
\begin{aligned}
& \bar{u}_{1}=B_{1} k_{11} e^{k_{11} x}+B_{2} k_{12} e^{k_{12} x}-B_{3} k_{11} e^{-k_{11} x}-B_{4} k_{12} e^{-k_{12} x} \\
& \bar{u}_{2}=-\left[k_{21} B_{5} e^{-k_{21} x}+k_{22} B_{6} e^{-k_{22} x}\right]
\end{aligned}
$$

where $B_{1}, B_{2}, B_{3}, B_{4}, B_{5}$ and $B_{6}$ are parameters that depend on $s$ only and $k_{i 1}, k_{i 2}$ are the roots with positive real parts of the characteristic equation

$$
\begin{aligned}
& \psi_{i} C_{t i}^{2} k^{4}-k^{2}\left[\psi_{i} s^{2}+C_{t i}^{2} \xi_{i} s^{2}+\varphi_{i} s^{2}\right]+\xi_{i} s^{4}=0 \\
& \bar{\theta}_{1}=\left(k_{11}^{2}-s^{2}\right)\left[B_{1} e^{k_{11} x}+B_{3} e^{-k_{11} x}\right]+\left(k_{12}^{2}-s^{2}\right)\left[B_{2} e^{k_{12} x}+B_{4} e^{-k_{12} x}\right] \\
& \bar{\theta}_{2}=\left(\psi_{2} k_{21}^{2}-\xi_{2} s^{2}\right) B_{5} e^{-k_{21} x}+\left(\psi_{2} k_{22}^{2}-\xi_{2} s^{2}\right) B_{6} e^{-k_{22} x}
\end{aligned}
$$

Substituting from equations (43), (46) into equation (39), we obtain

$$
\begin{aligned}
& \bar{\sigma}_{1}=s^{2}\left[{ }_{B_{1}} e^{k_{11} x}+B_{2} e^{k_{12} x}+B_{3} e^{-k_{11} x}+B_{4} e^{-k_{12} x}\right] \\
& \bar{\sigma}_{2}=\gamma \hat{i}_{2} s^{2}\left[B_{5} e^{-k_{21} x}+B_{6} e^{-k_{22} x}\right]
\end{aligned}
$$

Substituting from equations (45) into equation (40), we obtain the heat flux component $q_{i}$ in the form

$$
\begin{aligned}
& \bar{q}_{1}=-k_{11}\left(k_{11}^{2}-s^{2}\right)\left[B_{1} e^{k_{11} x}-B_{3} e^{-k_{11} x}\right]-k_{12}\left(k_{12}^{2}-s^{2}\right)\left[B_{2} e^{k_{12} x}-B_{4} e^{-k_{12} x}\right] \\
& \bar{q}_{2}=\alpha_{2}\left[k_{21}\left(\psi_{2} k_{21}^{2}-\xi_{2} s^{2}\right) B_{5} e^{-k_{21} x}+k_{22}\left(\psi_{2} k_{22}^{2}-\xi_{2} s^{2}\right) B_{6} e^{-k_{22} x}\right]
\end{aligned}
$$

Substituting from equations (43), (44), (47) and (48) into equations (25), (26) and (27), we obtain the following system of linear equations in the unknown parameters $B_{1}-B_{6}$

$$
\begin{aligned}
& B_{1}+B_{2}+B_{3}+B_{4}=0 \\
& \left(k_{11}^{2}-s^{2}\right)\left[B_{1}+B_{3}\right]+\left(k_{12}^{2}-s^{2}\right)\left[B_{2}+B_{4}\right]=\frac{\theta_{0}}{s} \\
& k_{11} B_{1} e^{k_{11} L}+k_{12} B_{2} e^{k_{12} L}-k_{11} B_{3} e^{-k_{11} L}-k_{12} B_{4} e^{-k_{12} L} \\
& \quad+k_{21} B_{5} e^{-k_{21} L}+k_{22} B_{6} e^{-k_{22} L}=0 \\
& \left(k_{11}^{2}-s^{2}\right)\left[B_{1} e^{k_{11} L}+B_{3} e^{-k_{11} L}\right]+\left(k_{12}^{2}-s^{2}\right)\left[B_{2} e^{k_{12} L}+B_{4} e^{-k_{12} L}\right] \\
& -\left(\psi_{2} k_{21}^{2}-\xi_{2} s^{2}\right) B_{5} e^{-k_{21} L}+\left(\psi_{2} k_{22}^{2}-\xi_{2} s^{2}\right) B_{6} e^{-k_{22} L}=0
\end{aligned}
$$


Citation: Khedr Mona El M, Khader SE (2017) A Problem in Thermoelasticity with and without Energy Dissipation. J Phys Math 8: 243. doi: 10.4172/2090-0902.1000243

Page 4 of 5

$$
\begin{aligned}
& B_{1} e^{k_{11} L}+B_{2} e^{k_{12} L}+B_{3} e^{-k_{11} L}+B_{4} e^{-k_{12} L} \\
& \quad-\gamma \xi_{2}\left[B_{5} e^{-k_{12} L}+B_{6} e^{-k_{22} L}\right]=0 \\
& k_{11}\left(k_{11}^{2}-s^{2}\right)\left[B_{1} e^{k_{11} L}-B_{3} e^{-k_{11} L}\right]+k_{12}\left(k_{12}^{2}-s^{2}\right)\left[B_{2} e^{k_{12} L}-B_{4} e^{-k_{12} L}\right] \\
& +\alpha_{2}\left[k_{21}\left(\psi_{2} k_{21}^{2}-\xi_{2} s^{2}\right) B_{5} e^{-k_{21} L}+k_{22}\left(\psi_{2} k_{22}^{2}-\xi_{2} s^{2}\right) B_{6} e^{-k_{22} L}\right]=0
\end{aligned}
$$

To obtain the inversion of the Laplace transform [7].

\section{Numerical Results and Discussion}

During numerical computations, the upper material was taken to be made of Carbon Steel, while that of the half-space was taken to be made of the Copper material. The constants of the problem were taken as shown in Table 1.

The computations were carried out for one values of time, namely for $t=0.2$. The temperature, displacement and stress distributions are shown in Figures 1-3, respectively. Solid line represents the case (GL theory), dotted line represents the case (GN theory). The FORTRAN programming language was used on a personal computer. The accuracy maintained was 4 digits for the numerical program.

We notice that the solution is non-zero only in a region of space adjacent to the upper surface. This region expands with the passage of time. This is different from the solutions obtained using either the uncoupled or the coupled theories of thermoelasticity where the

\begin{tabular}{|c|c|c|c|c|}
\hline$\rho_{1}=7833$ & $\alpha_{1}=1.474(10)^{-5}$ & $\mathrm{k}_{1}=54$ & $\mathrm{c}_{\mathrm{e} 1}=465$ & $\mathrm{~V}_{1}=6910112$ \\
\hline $\mathrm{I}_{1}=10.34(10)^{10}$ & $\mathrm{~m}_{1}=7.93(10)^{10}$ & $\mathrm{t}_{1}=0.02$ & $\mathrm{v}_{1}=0.02$ & $\mathrm{~T}_{1}=0.025$ \\
\hline$\rho_{2}=8954$ & $\mathrm{\alpha}_{2}=1.78(10)^{-5}$ & $\mathrm{k}_{2}=386$ & $\mathrm{c}_{\mathrm{e} 2}=383.1$ & $\mathrm{v}_{1}=0.025$ \\
\hline $\mathrm{I}_{2}=7.76(10)^{10}$ & $\mathrm{~m}_{2}=3.86(10)^{10}$ & $\mathrm{~V}_{2}=5518000$ & $\Psi_{2}=0.7399$ & $\xi_{2}=1.4315$ \\
\hline$\delta_{2}=0.48676$ & $\phi_{1}=0.01466$ & ${ }_{2}=0.001638$ & $\mathrm{~T}_{0}=293$ & \\
\hline
\end{tabular}

Table 1: The Material Constants.

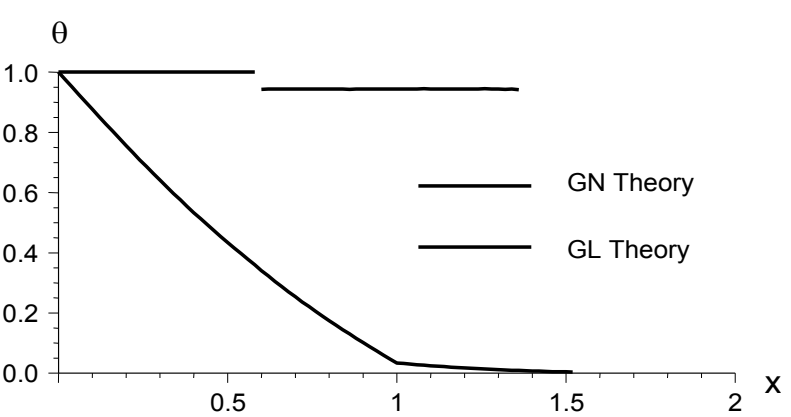

Figure 1: Temperature Distribution.

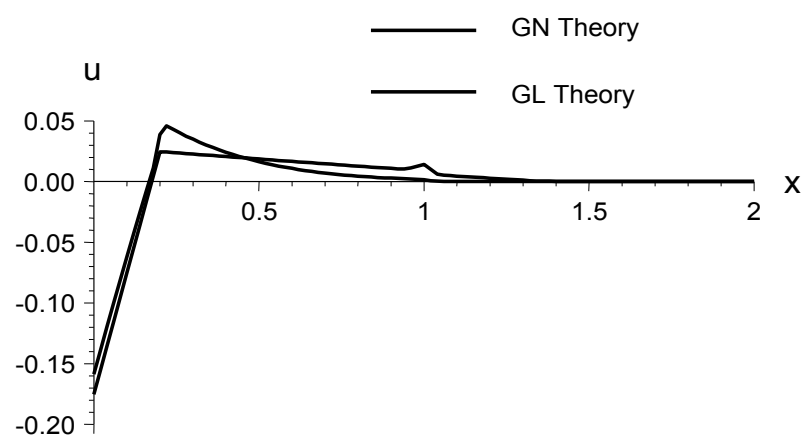

Figure 2: Displacement Distribution.

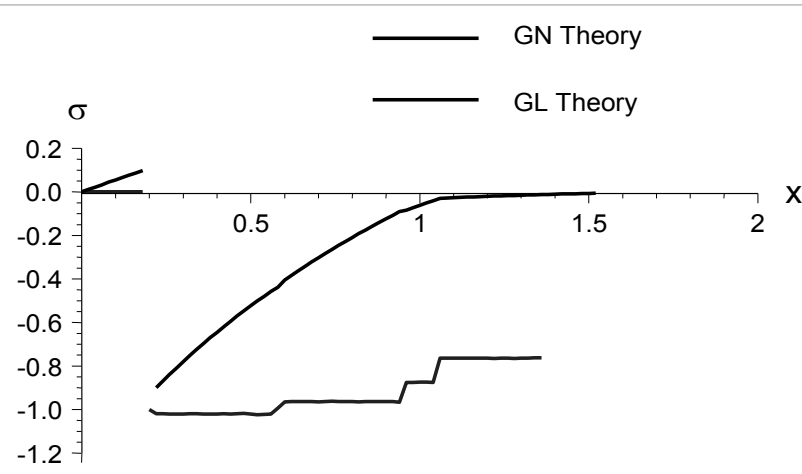

Figure 3: Stress Distribution.

solution is non-zero everywhere due to the infinite speed of wave propagation predicted by these theories. In Figure 1 we observed that the leading wave front has reached the point $\mathrm{x}=1.33$ for the two theories. The solutions are thus non-zero only in the region $0<\mathrm{X}<1.33$. The effects of the thermal shock have thus filled the region of the layer and reached the half-space in two theories. But in (GL theory), we fined that the dissipation in energy for the (GN theory) no dissipation in energy. In Figure 2, the value of displacement is bigger at the initial, and after that is decay to reached the zero at $x=1$ namely (GL theory), in (GN theory) the displacement reached to zero at $x=1.2$ namely. In Figure 3, we observed the discontinuity in the stresses at $x=0.2$ namely in both theories. In (GL theory), the lased of energy is observed. In all figures the beaver of all functions are different forms the two media but are continues.

\section{References}

1. Lord H, Shulman Y (1967) A Generalized Dynamical Theory of Thermoelasticity. J Mech Phys Solid 15: 299-309.

2. Sherief H, Anwar N (1992) Generalized Thermoelasticity Problem for a Plate Subjected To Moving Heat Sources On Both Sides. J Thermal Stresses 15 489-505.

3. Sherief H, Khader SE (2013) Propagation of discontinuities in electromagneto generalized thermoelasticity in cylindrical regions. Meccanica 48: 2511-2523.

4. Chen T, Jang H, Tseng A (1995) A. Transient thermal stresses in a multilayered anisotropic medium. ASME J Appl Mech117: 1067-1069.

5. Sharma JN, Kumar V Chand D (2003) Reflection of generalized thermoelastic waves from the boundary of a half-space. J Therm Stress 26: 925-942.

6. Elhagary MA (2010) A two-dimensional problem for two media in the generalized theory of thermoelasticity. J Therm Stress 33:993-1007.

7. Abd El-Latief AM, Khader SE (2015) Fractional Model of Thermoelasticity for a Half-Space Overlaid by a Thick Layer. Z Angew Math Mech 95: 511-518.

8. Sherief $\mathrm{H}$, El-Maghraby M (2009) A Thick Plate Problem in the Theory of Generalized Thermoelastic Diffusion. Int J Thermophysics 30: 2044-2057.

9. Green AE, Lindsay KA (1972) Thermoelasticity. J Elast 2: 1-7.

10. Sinha SB, Elsibai KA (1996) Reflection of thermoelastic waves at a solid halfspace with two thermal relaxation times. J Therm Stress 19: 763-777.

11. Sherief H (1992) Fundamental Solution for Thermoelasticity with Two Relaxation Times. Int J Engng Sci 30: 861-870.

12. Sherief H (1994) A Thermo-Mechanical Shock Problem for Thermoelasticity with Two Relaxation Times. Int J Engng Sci 32: 313-325.

13. Anwar N, Sherief H (1994) Boundary Integral Equation Formulation for Thermoelasticity with Two Relaxation Times. J Thermal Stresses 17: 257-270.

14. Song YQ, Zhang YC, Lu BH(2003) Transient Disturbance in a Half Space Under Thermoelasticity with Two Relaxation Times Due to Moving Internal Heat Source. Int J Thermophysics 24: 299-318. 
Citation: Khedr Mona El M, Khader SE (2017) A Problem in Thermoelasticity with and without Energy Dissipation. J Phys Math 8: 243. doi: 10.4172/2090-0902.1000243

Page 5 of 5

15. Green AE, Naghdi PM (1993) Thermoelasticity without energy dissipation. J Elast 31: 189-208.

16. Abd El-Latief AM, Khader SE (2014) Exact Solution of Thermoelastic Problem for a One-Dimensional Bar without Energy Dissipation .ISRN Mechanical Engineering, Hindwai.

17. Quintanilla R (1999) On the spatial behavior in thermoelasticity without energy dissipation. J Thermal Stresses 22: 213-224.
18. Quintanilla R (2002) on existence in thermoelasticity without energy dissipation. J Thermal Stresses 25: 195-202.

19. lesan $D(1998)$ On the theory of thermoelasticity without energy dissipation. J Thermal Stresses 21: 295-307.

20. Verma KL, Hasebe N (2001) Dispersion of thermoelastic waves in a plate with and without energy dissipation. International Journal of Thermophysics 22: 957-978.

21. Marin, M, Baleanu, D (2016) On vibrations in thermoelasticity without energy dissipation for micropolar bodies. Bound Value Probl, Springer. 